\title{
Understanding your syringe driver: patient information leaflet
}

\author{
Author: Kerran Kandola
}

\section{Aims}

The aim of this quality improvement project was to develop a new, up-to-date patient information leaflet about syringe drivers, tailored to the specific needs of patients and families.

\section{Methods}

A questionnaire was first designed to identify current understanding about syringe drivers and what additional information was needed. This consisted of six open answer questions and was distributed on the 15-bed inpatient unit at Willen Hospice between 1 September and 31 October 2017. Eligibility criteria included any patient being started on a syringe driver during admission, however a questionnaire was only given if appropriate and sensitive to do so.

Specific needs were identified based on the survey responses, and a new patient information leaflet was designed. This was reviewed by inpatient clinicians and an independent service user group, prior to approval by a clinical effectiveness group and being implemented across local inpatient and outpatient palliative care services.

\section{Results}

Nine sets of responses were obtained from patients starting drivers for either symptom management or end-of-life care. Fewer than half of those surveyed - four out of nine - were satisfied when a syringe driver was first proposed (denoted by responses of 'happy' or 'okay'). The remainder were less confident or indifferent with one patient specifically highlighting emotions of anxiety.

Our patients initially appeared to demonstrate a good basic understanding of syringe drivers. For example, five of the nine patients correctly identified their use for delivering medications over several hours to aid with symptom management and endof-life care. Nevertheless, when specifically asked what further information they would have preferred to have known, prior to the driver being started, significant gaps in understanding were revealed. This included how the driver would be maintained day-today, the practicalities of having a driver attached continuously, an awareness of drivers being used outside a clinical environment and which symptoms are commonly managed with syringe drivers.

\section{Conclusion}

Our patients have unmet needs surrounding syringe drivers with less than half feeling satisfied when they are started. This is likely multifactorial and may relate to an unclear understanding of syringe drivers, their benefits and existing preconceptions. Discussions alone are not always sufficient to provide adequate knowledge and allay underlying anxieties. The patient information leaflet designed here has been specifically tailored to address the knowledge gaps and concerns of our patients and will provide an invaluable aid to support future discussions.

\section{Conflict of interest statement}

The authors of this work declare no conflict of interest. 